\title{
Law of corresponding states for osmotic swelling of vesicles
}

\author{
Primož Peterlin, ${ }^{1, *}$ Vesna Arrigler, ${ }^{1}$ Emir Haleva, ${ }^{2}$ and Haim Diamant ${ }^{2}$, \\ ${ }^{1}$ Faculty of Medicine, Institute of Biophysics, University of Ljubljana, Lipičeva 2, 1000 Ljubljana, Slovenia \\ ${ }^{2}$ Raymond 8 Beverly Sackler School of Chemistry, Tel Aviv University, Tel Aviv 69978, Israel
}

\begin{abstract}
As solute molecules permeate into a vesicle due to a concentration difference across its membrane, the vesicle swells through osmosis. The swelling can be divided into two stages: (a) an "ironing" stage, where the volume-to-area ratio of the vesicle increases without a significant change in its area; (b) a stretching stage, where the vesicle grows while remaining essentially spherical, until it ruptures. We show that the crossover between these two stages can be represented as a broadened continuous phase transition. Consequently, the swelling curves for different vesicles and different permeating solutes can be rescaled into a single, theoretically predicted, universal curve. Such a data collapse is demonstrated for giant unilamellar POPC vesicles, osmotically swollen due to the permeation of urea, glycerol, or ethylene glycol. We thereby gain a sensitive measurement of the solutes' membrane permeability coefficients, finding a concentration-independent coefficient for urea, while those of glycerol and ethylene glycol are found to increase with solute concentration. In addition, we use the width of the transition, as extracted from the data collapse, to infer the number of independent bending modes that affect the thermodynamics of the vesicle in the transition region.
\end{abstract}

\section{INTRODUCTION}

Membrane vesicles are made of a closed bilayer of amphiphilic molecules in aqueous solution, having length scales of $0.1-100 \mu \mathrm{m}$. Used as simplified models of biological membranes, they have been one of the most extensively studied systems in soft-matter physics [1, 2]. Vesicles usually enclose both solvent (water) and solute molecules. Such vesicular capsules are ubiquitous in cell functions [3] and used as microreactors and delivery vehicles in various biomedical and cosmetic applications [4].

The hydrophobic core of the bilayer membrane poses a kinetic barrier to the permeation of water and watersoluble molecules into and out of the vesicle. Consequently, the permeability coefficients of various solutes across various membranes span a very wide range of values [5]. For example, the permeability coefficients of water, urea, and potassium ions through the membranes used in the current work are of the order, respectively, of $10^{2}, 10^{-2}$, and $10^{-8} \mu \mathrm{m} / \mathrm{s}[5]$. This implies that the time scales for permeation of water and the solutes examined here are well separated. Thus, the vesicles can safely be assumed to reside in a semi-permeable regime, in which their volume quickly (essentially immediately) re-adjusts through osmosis to a change in the number of enclosed solute molecules. As more solute molecules enter, therefore, the vesicle progressively swells - first approaching a spherical shape (the "ironing" stage), subsequently inflating as a sphere (the stretching stage), and eventually rupturing (osmotic lysis) [6].

In an earlier theoretical work we argued that a vesicle in such a semi-permeable regime should reach the end of the ironing stage (i.e., the maximum volume-to-area ratio) critically, through a continuous transition [7]. That

\footnotetext{
* Present address: Institute of Oncology, Zaloška 2, 1000 Ljubljana, Slovenia

$\dagger$ hdiamant@tau.ac.il
}

theory was restricted to unstretchable membranes. Here we extend the theory to the experimentally relevant case of stretchable membranes, finding a slightly modified but similar form of criticality. We then confirm the existence of the critical scaling behavior in experiments and utilize it to obtain a reliable measurement of permeability coefficients.

A crossover between two stages of vesicle swelling is well known in micropipette-aspiration experiments [8]. The stages are distinguished by a markedly different dependence of surface tension on surface strain - in the first stage the dependence is exponential, while in the second it is linear $[8-10]$. The essential difference between this scenario and the one addressed here lies in the different control parameters. In the former case the swelling is controlled by a hydrostatic pressure difference, whereas in the latter it is controlled by the number of encapsulated solute molecules. As a result, the two crossovers are not equivalent. As will be presented below, in fact, the monitored swelling of our vesicles, with its two distinct stages, occurs within the linear regime-i.e., the tension depends linearly on strain throughout the observed transition.

We begin by describing the experimental setup in Sec. III and the direct experimental results in Sec. [II] The analysis of the experimental data requires a revised theory, which is presented in Sec. IV In Sec. V we apply the theory to the experimental results to demonstrate data collapse onto a master curve - i.e., the law of corresponding states - and extract additional information, such as the permeability coefficients and the number of surface bending modes contributing to vesicle thermodynamics. Finally, we discuss the various results and their significance in Sec. VI 


\section{EXPERIMENTAL SETUP}

D-(+)-glucose, D- $(+)$-sucrose, glycerol, urea, and ethylene glycol were purchased from Fluka (Buchs, Switzerland). Methanol and chloroform were purchased from Kemika (Zagreb, Croatia). 1-palmitoyl-2-oleoyl-snglycero-3-phosphocholine (POPC) was purchased from Avanti Polar Lipids (Alabaster, USA). All the solutions were prepared in double-distilled sterile water.

A suspension of POPC giant unilamellar vesicles (GUVs) in 0.1 or $0.2 \mathrm{~mol} / \mathrm{L} 1: 1$ sucrose/glucose solution was prepared using an electroformation method, described in Ref. 11 with some modifications [12, 13]. Lipids were dissolved in a mixture of chloroform/methanol $(2: 1$, $\mathrm{v} / \mathrm{v}$ ) to a concentration of $1 \mathrm{mg} / \mathrm{mL}$. A volume of $25 \mu \mathrm{L}$ of the lipid solution was spread onto a pair of Pt electrodes and dried under reduced pressure (water aspirator; $\approx 60 \mathrm{mmHg}$ ) for 2 hours. The electrodes were then placed into an electroformation chamber, which was filled with 0.1 or $0.2 \mathrm{~mol} / \mathrm{L}$ sucrose. AC current $(8 \mathrm{~V}, 10 \mathrm{~Hz})$ was applied, and the voltage and frequency were reduced in steps to the final values of $1 \mathrm{~V}$ and $1 \mathrm{~Hz}$ [13]. Subsequently, the chamber was first drained into a beaker and then flushed with an equal volume of isomolar glucose solution, thus resulting in a suspension of GUVs containing entrapped sucrose in a 1:1 sucrose/glucose solution, which increases the contrast in a phase contrast setup and facilitates vesicle manipulation [14]. This procedure yields mostly spherical unilamellar vesicles, with diameters of up to $100 \mu \mathrm{m}$.

An inverted optical microscope (Nikon Diaphot 200, objective 20/0.40 Ph2 DL) with micro-manipulating equipment (Narishige MMN-1/MMO-202) and a cooled CCD camera (Hamamatsu ORCA-ER; C4742-9512ERG), connected via an IEEE-1394 interface to a PC running Hamamatsu Wasabi software, was used to obtain phase contrast micrographs. In the streaming mode, the camera provides $1344 \times 1024$ 12-bit grayscale images at a rate of 8.9 images/s.

In the experiment, an individual spherical GUV is selected, fully aspirated into a glass micropipette whose inner diameter exceeds the vesicle's diameter, and transferred from a solution containing solutes of very low membrane permeability (1:1 glucose/sucrose) into an isoosmolar solution of a more permeable solute (glycerol, urea, or ethylene glycol), where the content of the micropipette is released, and the micropipette is subsequently removed. Vesicle response is recorded using a CCD camera mounted on the microscope. The radius of the vesicle's cross-section was determined from the recorded series of images using a least-squares procedure [15].

\section{EXPERIMENTAL RESULTS}

Once the vesicle is released and gets in contact with the target solution, a transient increase of its cross-sectional radius, $R_{1}$, is observed. We attribute it to a small hypotonicity of the outer solution, causing the vesicle to slightly deflate and change its shape from a sphere into an oblate spheroid [16, 17]. Gravity should lead to a small deviation of the shape from a perfect oblate spheroid [16], which we neglect here. A more important effect of gravity is the breaking of the problem's rotational symmetry, such that the observed lateral radius, $R_{1}$, is always the spheroid's larger radius. On a longer time scale than the transient deflation, the vesicle inflates due to solute permeation and the accompanying osmosis (i.e., water influx). This causes the vesicle to become more spherical, making the observed $R_{1}$ decrease. (See Fig. 11) This is the ironing stage, where the swelling increases the volume-to-area ratio. After a certain time, $R_{1}$ reaches a minimum and starts increasing. (See again Fig. 11) This marks the crossover to the stretching stage, where the vesicle continues to swell essentially as an inflating sphere. When the membrane reaches a critical strain, it ruptures, and the vesicle bursts. Subsequently, the membrane is resealed and another cycle of swelling commences. The repeated burst-swelling cycles were analyzed elsewhere [13]. In the present work we focus on the swelling that precedes the first burst.

The ironing and stretching stages are clearly distinguished in Fig. 11 as the decreasing and increasing parts of the swelling curve, respectively. To have a systematic definition of the time axis for all vesicles we define $t=0$ at the minimum of the curve. We associate the radius at that minimum, $R_{0}$, with the relaxed area of the vesicle, $A_{0} \equiv 4 \pi R_{0}^{2}$. (We return to examine this assumption in Sec.VI.) From each such curve we also extract the radius at rupture, $R_{\text {rup }}$. This yields a direct measurement of the membrane's rupture strain,

$$
\epsilon_{\text {rup }}=\left(R_{\text {rup }} / R_{0}\right)^{2}-1 .
$$

Figures 2(a)-(c) show the swelling curves measured for POPC vesicles of broadly distributed sizes due to the permeation of three different solutes: urea, glycerol, and ethylene glycol. Each of these curves has the typical shape shown in Fig. 1, yet the polydispersity of the vesicles flattens them once they are displayed together. This is demonstrated in Fig. 3, where we have replotted the swelling curves for urea after rescaling the ordinate of each of them by its minimum radius, $R_{0}$. Figure 3 shows that such a simple size rescaling does not collapse the data onto a master curve. A more detailed analysis of the temporal swelling curves requires a theory, which is presented in the next section. In Fig. 2 it is apparent, nonetheless, that there is no correlation between the transition width (sharpness of the curve's minimum) and the vesicle size. We return to this point in Sec. $\mathrm{V}$.

A dataset that can be presented without further analysis is the one for $\epsilon_{\text {rup }}$, the critical surface strain at rupture, calculated from the directly measured values of $R_{0}$ and $R_{\text {rup }}$ according to Eq. (11). Since rupture is a result of pore formation, which is a nucleated event [18, 19], the critical strain as measured in individual vesicles is 


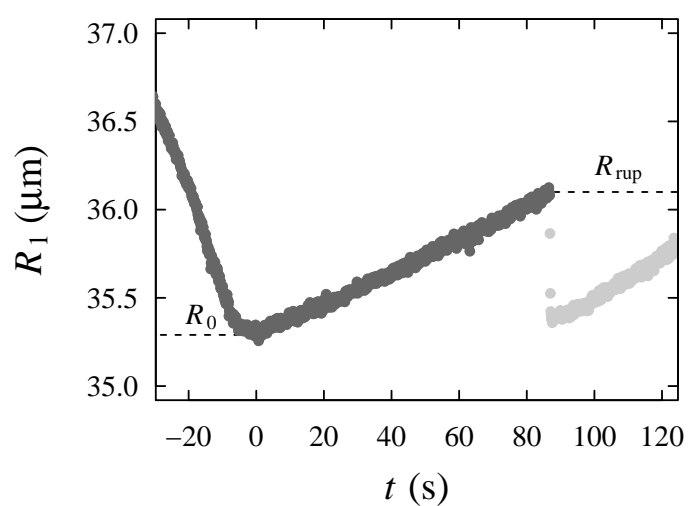

FIG. 1. Typical swelling curve, showing the change in the observed (larger) radius of an oblate spheroidal POPC vesicle as a function of time due to inward permeation of urea. The decreasing part of the curve corresponds to the ironing stage, and the increasing one to the stretching stage. The minimum of the curve is defined as $\left(t=0, R_{1}=R_{0}\right)$. The present analysis concerns the swelling up to the point of first rupture $\left(R_{1}=R_{\text {rup }}\right)$.

a stochastic variable. Figure 4 shows the histogram of measured rupture strains. From this distribution we get $\epsilon_{\text {rup }}=0.055 \pm 0.02$, i.e., about 5 percent, which agrees with known values for the maximum sustainable strain of lipid bilayers [8].

\section{THEORY}

Models of vesicles usually focus on the statistical mechanics of the membrane under the appropriate constraints, the starting point being the Helfrich Hamiltonian [1, 2, 9, 10, 16]. In the current work we choose a different, more general description, based on the thermodynamics of small systems [20]. This is because the phenomenon of main interest here - the transition between the ironing and stretching stages - arises from competition between volume and surface effects, which is independent of the detailed interactions. Since the transition is of a mean-field type [7], such a thermodynamic description should be sufficient.

We consider a dilute solution enclosed in a membrane vesicle. This system is in thermal and pressure contact with a much larger solution having temperature $T$ and pressure $p_{\mathrm{o}}$. The system is at thermodynamic equilibrium. Thus, its temperature is $T$. However, since we deal with strong surface effects, the system's equilibrium pressure may deviate from the outer pressure $p_{\mathrm{o}}$. The enclosed solution contains $Q$ solute molecules 1 Although

\footnotetext{
1 In the experiment described above the vesicle actually contains two solutes - one that permeates the membrane and another that is trapped inside the vesicle. Analysis for an ideal solution,
}

$Q$ in the experiment changes with time, we assume, because the solute has much lower permeability than water, that the system is at all times in quasi-equilibrium, given the instantaneous number of enclosed solute molecules, $Q(t)$. Due to the fast water exchange, we treat both the volume $V$ and surface area $A$ of the vesicle as free, independent thermodynamic variables. Alternatively, one can define a reduced volume,

$$
v(V, A)=6 \sqrt{\pi} \frac{V}{A^{3 / 2}} \in(0,1),
$$

which characterizes the deviation of the vesicle from a perfect spherical shape, and consider $v$ and $A$ as the thermodynamic variables.

We divide the Gibbs free energy of the system into three-dimensional (volume) and two-dimensional (surface) contributions:

$$
G=G_{3 \mathrm{D}}+F_{2 \mathrm{D}}
$$

Taking the enclosed solution to be ideal and dilute, we write its Gibbs free energy as

$$
G_{3 \mathrm{D}}=k_{\mathrm{B}} T Q\left[\ln \left(\frac{Q v_{\mathrm{w}}}{V}\right)-1\right]+p_{\mathrm{o}} V,
$$

where $v_{\mathrm{w}}$ is the molecular volume of water.

As to the surface, we distinguish between in-plane (stretching) and out-of-plane (bending) degrees of freedom, and assume that the vesicle is sufficiently large such that the two are decoupled, i.e., contribute additively to the Helmholtz free energy,

$$
F_{2 \mathrm{D}}=F_{\mathrm{s}}+F_{\mathrm{b}}
$$

We further assume that the membrane's in-plane free energy is minimum for a certain relaxed area, $A_{0}$, and expand it to leading order about that minimum,

$$
F_{\mathrm{s}}=\frac{K}{2 A_{0}}\left(A-A_{0}\right)^{2}
$$

where $K$ is the membrane's stretching modulus. In the bending free energy we include only the contribution from undulation entropy, which, assuming small fluctuations about a spherical shape, is given by [7]

$$
F_{\mathrm{b}}=-\frac{1}{2} N k_{\mathrm{B}} T \ln (1-v),
$$

where $N$ is the total number of independent bending modes contributing to the membrane's thermodynamics. Equation (7), which describes the suppression of bending fluctuations as the vesicle approaches the maximum volume-to-area ratio $(v \rightarrow 1)$, is the only statisticalmechanical input to the theory. It is analogous to an

containing $Q_{1}$ molecules of the former and $Q_{2}$ molecules of the latter, leads to identical results once we take $Q=Q_{1}+Q_{2}$. 

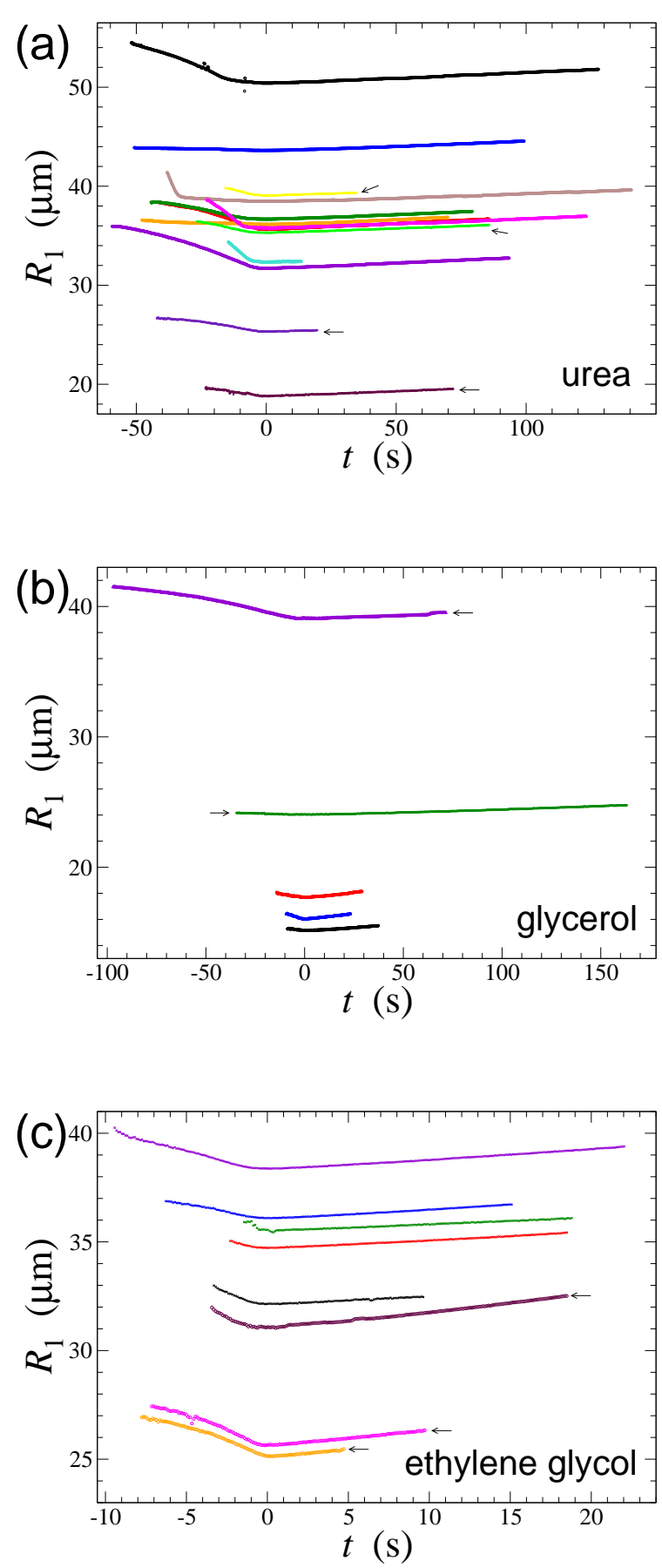

FIG. 2. Swelling curves for POPC vesicles under the permeation of (a) urea, (b) glycerol, and (c) ethylene glycol. Outer solute concentrations are $0.1 \mathrm{M}, 0.2 \mathrm{M}$ (left-pointing arrows), and $0.11 \mathrm{M}$ (right-pointing arrow in (b)).

equipartition principle; here each bending mode contributes an equal amount of $\left(k_{\mathrm{B}} T / 2\right)|\ln (1-v)|$ to the free energy [7]. Thus, this expression holds quite generally for nearly spherical vesicles, regardless of their detailed properties. Equation (7) also provides the crucial coupling between $G_{3 \mathrm{D}}$ and $F_{2 \mathrm{D}}$, since $v$ depends on both

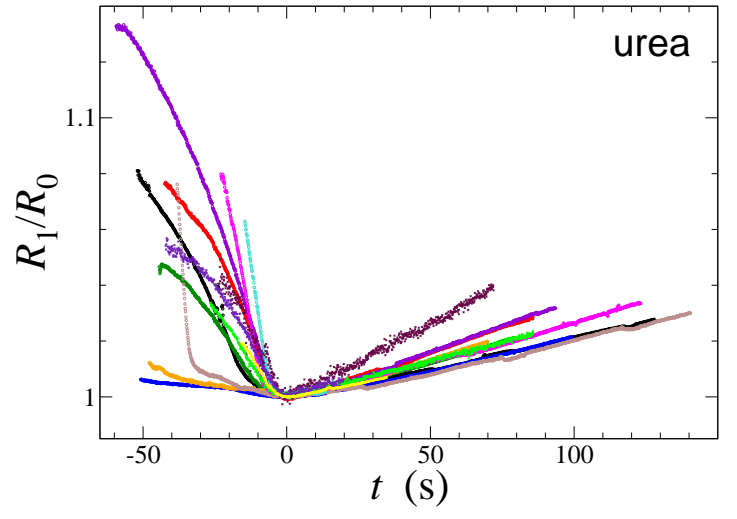

FIG. 3. The swelling curves of Fig. 2(a) replotted using the same colors after being rescaled by their minima.

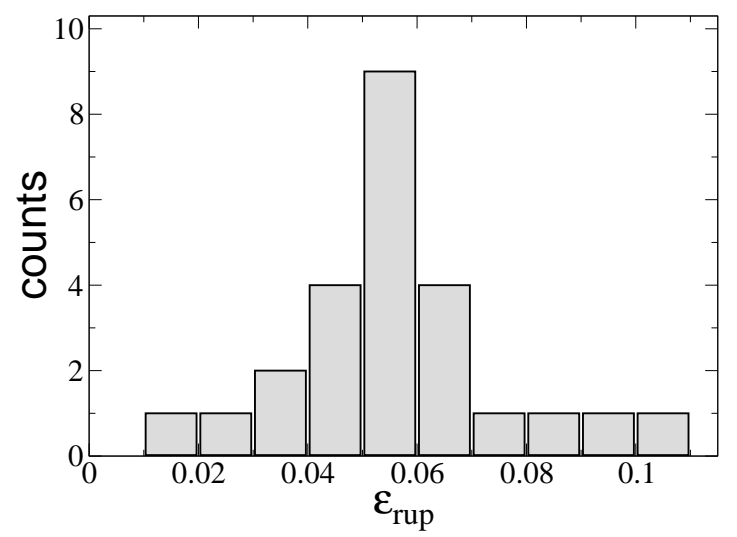

FIG. 4. Histogram of measured rupture strains for POPC vesicles. The distribution has a mean of 0.055 and standard deviation of 0.02 .

$V$ and $A$ according to Eq. (2).

The number of modes affecting the thermodynamics, $N$, which should be proportional to the number of lipid molecules in the membrane (i.e., to the membrane's relaxed area $A_{0}$ ), is a priori unknown. Further discussion of this number, its dependence on membrane's parameters, and its link to other theoretical treatments of membrane fluctuations, is deferred to Sec. VI. Here we prefer to leave it unspecified and extract it from the experiment. The number of modes is used to define an intensive area, or effective patch size, $a \equiv A / N$, having the relaxed value $a_{0} \equiv A_{0} / N$.

One could add, of course, various other contributions to the free energy of the vesicle, such as the membrane's bending energy, surface-surface and surface-solute interactions (e.g., electrostatic ones), gravitational energy, etc.. All these, however, do not significantly change when the vesicle becomes spherical and begins to stretch. In other words, in the limit (to be studied below) where $(1-v)$ becomes singular, these contributions are non- 
singular, and, hence, cannot affect the critical behavior in the transition region.

Equations (3) - (7) define the Gibbs free energy of our finite-size system as a function of $\left(T, p_{\mathrm{o}}, Q, N\right)$ and also $V$ and $A$. The equilibrium free energy as a function of $\left(T, p_{\mathrm{o}}, Q, N\right)$ alone is obtained by minimizing $G$ with respect to the volume and area of the vesicle. Note that by using this procedure we circumvent altogether the subtle and controversial issue of membrane tension. (See, e.g., Refs. 20 23 and references therein.) The Laplace tension and surface pressure of the membrane emerge naturally from the minimization with respect to $V$ and $A$, respectively. That is also the reason why we have formulated the Helmholtz (rather than Gibbs) free energy of the surface, to avoid specifying any thermodynamic variables pertaining to the surface other than $T, A$, and $N$. Another related subtlety is that $K=A\left(\partial^{2} F_{2 \mathrm{D}} / \partial A^{2}\right)$ at fixed $v$ (rather than fixed $V$ ), otherwise there would be another contribution to the stretching modulus from changes in the volume-to-area ratio.

The minimization leads to the following two equations:

$$
\begin{aligned}
\frac{1}{2} \delta_{N} \frac{v}{1-v}+\left(\frac{a}{a_{0}}\right)^{3 / 2} v & =\frac{Q}{Q_{\mathrm{c}}} \\
\frac{2 \delta_{N}}{3 \delta_{K}} \frac{a\left(a-a_{0}\right)}{a_{0}^{2}}+\left(\frac{a}{a_{0}}\right)^{3 / 2} v & =\frac{Q}{Q_{\mathrm{c}}},
\end{aligned}
$$

from which one can calculate $v$ and $a$. In Eqs. (8) and (9) we have defined

$$
Q_{\mathrm{c}} \equiv \frac{p_{\mathrm{o}} V_{0}}{k_{\mathrm{B}} T}, \quad V_{0} \equiv \frac{A_{0}^{3 / 2}}{6 \sqrt{\pi}},
$$

along with two small parameters,

$$
\delta_{N} \equiv \frac{N}{Q_{\mathrm{c}}} \sim N^{-1 / 2}, \quad \delta_{K} \equiv \frac{k_{\mathrm{B}} T}{K a_{0}} \sim K^{-1},
$$

the first related to the vesicle's finite size, and the other to the membrane's finite stretchability.

Before proceeding, it is instructive to examine Eqs. (8) and (9) in two limits. The first is the ordinary thermodynamic limit of infinite system size $(N \rightarrow \infty$, $\delta_{N} \rightarrow 0$ ) while keeping the stretching modulus finite. In this case Eqs. (8) and (9) become degenerate and yield the expected equilibration of inner and outer pressures, $k_{\mathrm{B}} T Q / V=p_{\mathrm{o}}$. The second limit is that of an unstretchable membrane $\left(K \rightarrow \infty, \delta_{K} \rightarrow 0\right)$. In this case Eq. (9) imposes $a=a_{0}$, and Eq. (8) then yields the behavior that was studied in Ref. 7, with criticality of $v$ as $N \rightarrow \infty$. Outside these two limits we get from these equations the relation

$$
\delta_{K} \frac{v}{1-v}=\frac{4 a\left(a-a_{0}\right)}{3 a_{0}^{2}},
$$

which demonstrates the actual interplay between the volume-to-area ratio and surface strain. We see that the vesicle can never attain a perfect spherical shape $(v=1)$, because this would require unphysical strain $(a \rightarrow \infty)$. The deviation of the area from its relaxed value is inversely proportional to the deviation of the shape from a perfect sphere. This relation becomes more sensitive the smaller the value of $\delta_{K}-i . e$. , the vesicle should be strongly swollen, $1-v \sim \delta_{K} \ll 1$, to get an appreciable strain $\left(a-a_{0}\right) / a_{0}$.

To present the detailed behavior of the transition, we define a control parameter $q$, proportional to the number of enclosed solute molecules, and an order parameter $M$, measuring the deviation from a spherical shape,

$$
q \equiv Q / Q_{\mathrm{c}}-1, \quad M \equiv 1-v .
$$

Solving Eqs. (8) and (9) in the vicinity of the transition, we get

$$
\begin{aligned}
& M(q)=\Delta \tilde{M}(q / \Delta), \quad \Delta=\left[2 \delta_{N}+(9 / 2) \delta_{K}\right]^{1 / 2} \\
& \tilde{M}(x)=\left(\sqrt{1+x^{2}}-x\right) / 2 .
\end{aligned}
$$

Thus, as $q$ increases from negative to positive values, $M$ crosses over from appreciable values, $M \simeq|q|$, to very small ones, $M \simeq \Delta /(4 q)$. The crossover occurs over a range of $q$ defined by $\Delta$. In the limit $\Delta \rightarrow 0$ the point $(q=0, M=0)$ becomes a singular corner where $d M / d q$ undergoes a discontinuous jump. The finite size of the vesicle and the stretchability of the membrane both contribute to the broadening of the transition. If we take $\delta_{K}=0$, Eq. (14) properly coincides with the critical behavior in the case of an unstretchable membrane [7]. Which of the two broadening effects dominates in practice is determined by the ratio

$$
\frac{\delta_{N}}{\delta_{K}} \sim \frac{K / R_{0}}{p_{\mathrm{o}}}
$$

where the right-hand side reflects the competition between surface (numerator) and volume (denominator) effects.

Substituting Eq. (14) in Eq. (12), we obtain for the surface strain, $\epsilon \equiv\left(a-a_{0}\right) / a_{0}=(3 / 4) \delta_{K} M^{-1}$. Yet, since the scaling function satisfies $[\tilde{M}(x)]^{-1}=4 \tilde{M}(-x)$, we can rewrite this result as

$$
\epsilon(q)=\frac{2}{3\left[1+(4 / 9)\left(\delta_{N} / \delta_{K}\right)\right]} \Delta \tilde{M}(-q / \Delta) .
$$

Thus, up to a prefactor, the strain's behavior is a mirror image of that of $M$. As $q$ increases from negative to positive values, $\epsilon$ changes from very small values, $\sim \Delta /|q|$, to appreciable ones, $\sim q$. Similarly, combining Eqs. (8), (12), and (14), we get for the Laplace tension,

$$
\gamma(q)=\frac{R}{2}\left(\frac{k_{\mathrm{B}} T Q}{V}-p_{\mathrm{o}}\right) \simeq \frac{3 k_{\mathrm{B}} T}{4 a_{0}}[M(q)]^{-1}=K \epsilon(q),
$$

i.e., the surface tension follows the same behavior as the strain, becoming appreciable only for sufficiently large, positive $q$. Equation (16) implies that within the transition region the tension is in the so-called linear regime 
[8, 9], where it is proportional to the surface strain. Substituting the rupture strain found above, $\epsilon_{\text {rup }} \simeq 0.05$, and $K \simeq 240 \mathrm{mN} / \mathrm{m} \mathrm{24}$, we obtain a rupture tension of order $10 \mathrm{mN} / \mathrm{m}$, which is in line with previously reported values of $5-10 \mathrm{mN} / \mathrm{m}$ 8].

Equation (14) [equivalently, (15) or (16)] describes a law of corresponding states for osmotically swollen, nearly spherical vesicles. It predicts that, upon rescaling, the swelling (i.e., volume-to-area ratio, strain, or tension) of such vesicles, regardless of their size, composition, and the nature or concentration of solute, could be collapsed onto a single universal curve defined by the function $\tilde{M}(x)$. Thus, despite the many physical parameters affecting the process, only two parameters actually suffice to completely characterize the osmotic swelling of any nearly spherical vesicle - the location of the transition, $Q_{\mathrm{c}}$, and its width, $\Delta$.

An interesting consequence of the competition between surface and volume effects is found when we examine the vesicle's swelling beyond the transition, well into the stretching stage. In this case, $q \gg \Delta$, Eq. (15) becomes

$$
\epsilon=\frac{2}{3} \frac{q}{1+(4 / 9) \delta_{N} / \delta_{K}}=\frac{2}{3} \frac{k_{\mathrm{B}} T Q / V_{0}-p_{\mathrm{o}}}{p_{\mathrm{o}}+(4 / 3) K / R_{0}} .
$$

Upon replacing the surface strain of a spherical vesicle with its relative volume change, $\left(V-V_{0}\right) / V_{0}=(3 / 2) \epsilon$, and the outer pressure of a dilute solution with its concentration, $p_{\mathrm{o}}=k_{\mathrm{B}} T c_{\mathrm{o}}$, Eq. (17) reproduces a known result [25], which was used in earlier analyses of the stretching stage [13, 25]. As we have seen above, if $\delta_{N} / \delta_{K} \ll 1$, the finite stretching modulus determines the transition width $\Delta$ [Eq. (14)]. At the same time, according to Eq. (17), the relatively small value of $K$ in this case makes the swelling beyond the transition insensitive to $K$. Being governed by volume effects, the growth in the stretching stage is then simply proportional to the increasing number of enclosed molecules [13].

\section{DATA ANALYSIS}

Let us first check which of the broadening factorsfinite size or stretchability - is dominant in the experiment. The stretching modulus of a POPC bilayer is $K \simeq 240 \mathrm{mN} / \mathrm{m} \mathrm{[24]}$. Our vesicles have $R_{0} \sim 20-50 \mu \mathrm{m}$, and the outer concentration is $c_{\mathrm{O}} \sim 0.1-0.2 \mathrm{M}$. We get $\delta_{N} / \delta_{K} \sim K /\left(R_{0} k_{\mathrm{B}} T c_{\mathrm{o}}\right) \sim 0.01-0.1$. Hence, the transition width is governed by the finite stretching modulus and is expected, therefore, to be independent of vesicle size,

$$
\Delta \simeq\left(\frac{9}{2} \delta_{K}\right)^{1 / 2}=\left(\frac{9 k_{\mathrm{B}} T}{2 K a_{0}}\right)^{1 / 2} .
$$

As noted above, this implies also that the stretching stage following the transition is insensitive to the value of $K$, with $\epsilon(q \gg \Delta) \simeq(2 / 3) q$.
To further check the theoretical predictions of the preceding section, we need to relate them to the experimental observables demonstrated in Fig. 1-i.e., the temporal change in the principal radius of an oblate spheroidal vesicle, $R_{1}(t)$.

We begin with the time axis. The membrane permeability coefficients of the examined solutes are of order $P \sim 10^{-2} \mu \mathrm{m} / \mathrm{s}$ [5, 13, 26], the vesicles are of radii $R_{0} \sim 20-50 \mu \mathrm{m}$, and the swelling process lasts about $t \sim 10-100 \mathrm{~s}$. We have $\left(P / R_{0}\right) t \sim 0.001-0.05$, implying that the concentration of permeating solute inside the vesicle never exceeds a few percent of its outer concentration. Hence, throughout the observed swelling we may assume $d Q / d t=P A_{0} c_{\mathrm{o}}$, leading to

$$
q=\left(3 P / R_{0}\right) t+\text { const. }
$$

Thus, the time axis, up to a linear transformation that depends on vesicle size, is equivalent to our control parameter.

Next, we should adapt the theoretical scaling relations, Eqs. (14) and (15), so that they could be applied to the measured swelling curves, $R_{1}(t)$. This calculation is presented in the Appendix. Given the experimentally measured dependence, $\left[R_{1}(t) / R_{0}\right]^{3}-1 \equiv g_{\exp }(t)$, we find that the following scale and shift transformation:

$$
\begin{aligned}
f(x)= & (15 \Delta / 8)^{-1 / 2}\left\{g_{\exp }\left[x-\left(15^{1 / 3} / 4\right) \Delta^{-1 / 3}\right]-x \Delta\right. \\
& \left.+3\left(15^{1 / 3} / 4\right) \Delta^{2 / 3}\right\},
\end{aligned}
$$

where $x=q / \Delta$ and $q=\left(3 P / R_{0}\right) t$, should collapse the data onto the universal function

$$
f(x)=\left(\sqrt{1+x^{2}}-x\right)^{1 / 2} .
$$

The rescaling scheme defined in Eq. (20), which has been dictated by the nature of the experiment, is not as elegant as the theoretical law of corresponding states, Eq. (14). Nevertheless, the two are equivalent and similarly straightforward. We have applied the scheme to the data of Fig. 2 while using the permeability $P$ (which merely scales the horizontal axis) and the transition width $\Delta$ as two fitting parameters. The results are presented in Fig. 5. showing successful data collapse, within the transition region and above it, onto the predicted universal curve, Eq. (21). Theoretically, successful data collapse is expected in the vicinity of the transition. We obtain data collapse also above the transition, throughout the stretching stage, for technical reasons explained in the Appendix. Below the transition (i.e., for sufficiently negative $x=q / \Delta$ ), the different swelling curves in Fig. [5] depart from the universal behavior. The entropy effects responsible for this departure, which set in at sufficiently low surface tension, will be described in Sec. VI.

In Fig. 6] we present the fitted values for the POPCmembrane permeability coefficients of the three examined solutes. The values for the permeability of urea [Fig. 6(a)] are narrowly distributed and concentrationindependent, yielding $P=0.013 \pm 0.001 \mu \mathrm{m} / \mathrm{s}$. This 

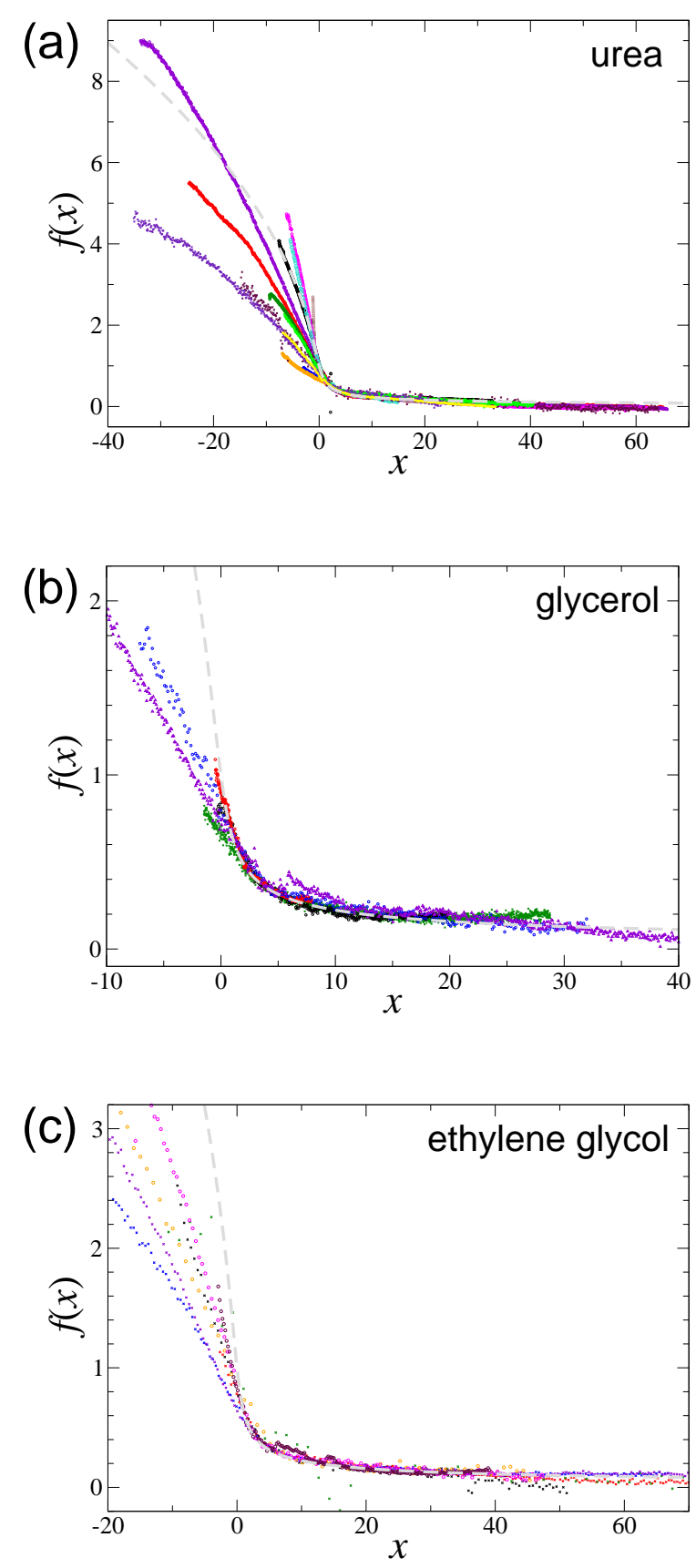

FIG. 5. The experimental data of Fig. 2 replotted using the same colors after being transformed according to Eq. (20). The dashed gray line shows the theoretical master curve, $f(x)=\left(\sqrt{1+x^{2}}-x\right)^{1 / 2}$.

agrees well with the result of $0.014 \mu \mathrm{m} / \mathrm{s}$, obtained for $0.1 \mu \mathrm{m}$-radius DOPC vesicles by Paula et al. [5] using dynamic light scattering, despite the different phospholipid and the large difference in the sizes of the studied systems. For glycerol we get values that significantly increase with glycerol concentration [Fig. 6(b)]: $P=0.0053,0.0074$, and $0.019 \pm 0.006 \mu \mathrm{m} / \mathrm{s}$ for $c_{\mathrm{o}}=0.1$, 0.11 , and $0.2 \mathrm{M}$, respectively. The last value agrees with the value of $0.021 \pm 0.008 \mu \mathrm{m} / \mathrm{s}$, extracted for the same vesicles, at $c_{\mathrm{o}}=0.2 \mathrm{M}$ glycerol, from a different analysis of the stretch-burst cycles that follow vesicle rupture 13]. A glycerol permeability of $0.027 \mu \mathrm{m} / \mathrm{s}$ was reported for $0.1 \mu \mathrm{m}$-radius DOPC vesicles by Paula et al. [5] and by Dordas and Brown [26], both at $c_{\mathrm{o}}=0.4 \mathrm{M}$. These results are not inconsistent with ours, especially given the concentration dependence. For ethylene glycol we obtain more broadly distributed values that depend on concentration [Fig. 6(b)]: $P=0.046 \pm 0.006$ and $0.085 \pm 0.01$ $\mu \mathrm{m} / \mathrm{s}$ for $c_{\mathrm{o}}=0.1$ and $0.2 \mathrm{M}$, respectively. The only previously measured permeability for ethylene glycol that we are aware of is $0.88 \mu \mathrm{m} / \mathrm{s}$, obtained by Orbach and Finkelstein [27] using conductivity measurements for a flat egg-PC membrane. This value is larger than ours by an order of magnitude. We note, though, the very different conditions under which the two experiments were conducted. All permeability values reported here, including the concentration-dependent ones for glycerol and ethylene glycol, are consistent with those found from the alternative analysis of stretch-burst cycles (unpublished results).
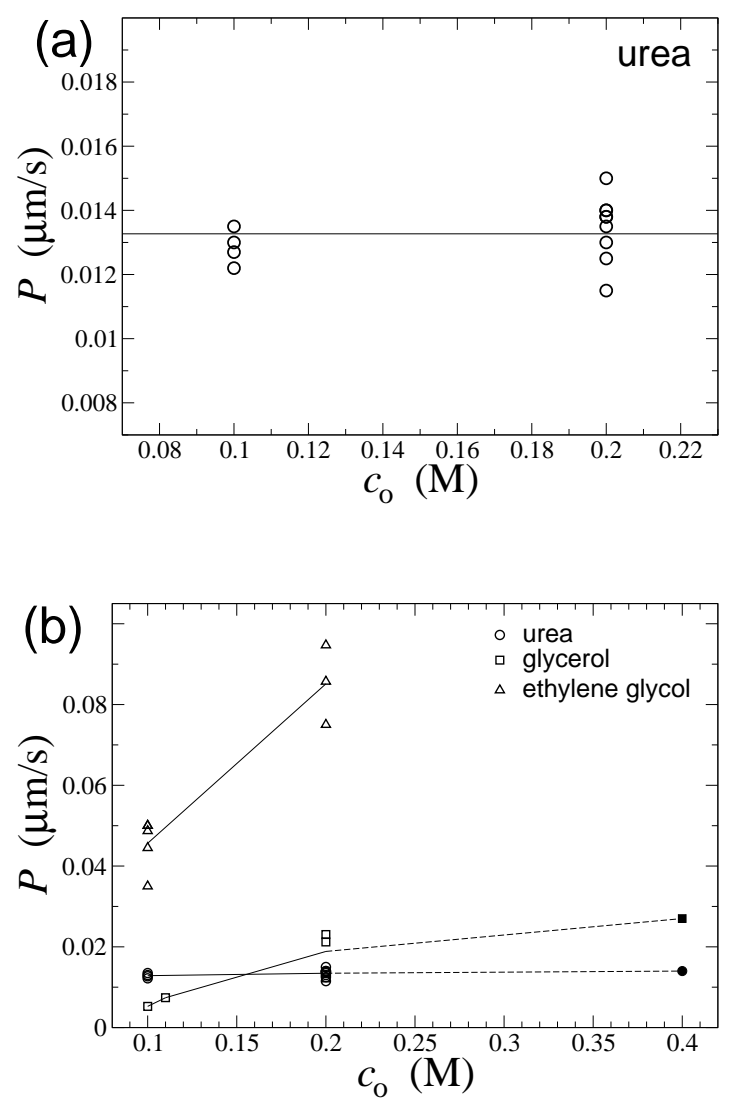

FIG. 6. Permeability coefficients as a function of outer solute concentration for urea (a) and all three solutes (b). Uncertainties in individual fitted values are smaller than the scatter for different vesicles. Filled symbols at $c_{\mathrm{o}}=0.4 \mathrm{M}$ are results for DOPC vesicles taken from Ref. 5 . The lines are guides to the eye. 
We now turn to the second fitting parameter - the transition width, $\Delta$. It is found to lie in the range $10^{-3}$ $10^{-2}$ and be uncorrelated with the vesicle size (Fig. 7), as predicted above. The small values of $\Delta$ establish the selfconsistency of our analysis for the crossover between the two swelling stages as a slightly broadened phase transition, and thus explain the success of the resulting data collapse. The rather broad distribution of these values imply that the transition width is affected by a stochastic variable. Of the three parameters appearing in Eq. (18) - $-T, K$, and $a_{0}$ - only the effective patch size $a_{0}$ may be responsible for such stochasticity. In Fig. 7(b) we show the distribution of patch sizes, as arising from Eq. (18) using the measured $\Delta$ and $K=240 \mathrm{mN} / \mathrm{m}$. The mean patch size is $a_{0} \simeq 0.01 \mu \mathrm{m}^{2}$, which is much smaller than the vesicle area, but much larger than the molecular size. Such a patch contains about $10^{4}-10^{5}$ lipids. In the inset of Fig. 7(b) we recast the same data in terms of the number of effective bending modes, $N=A_{0} / a_{0}$. The number of modes is broadly distributed in the range $10^{5}$ $10^{7}$, making its increasing trend with vesicle area hardly discernible.
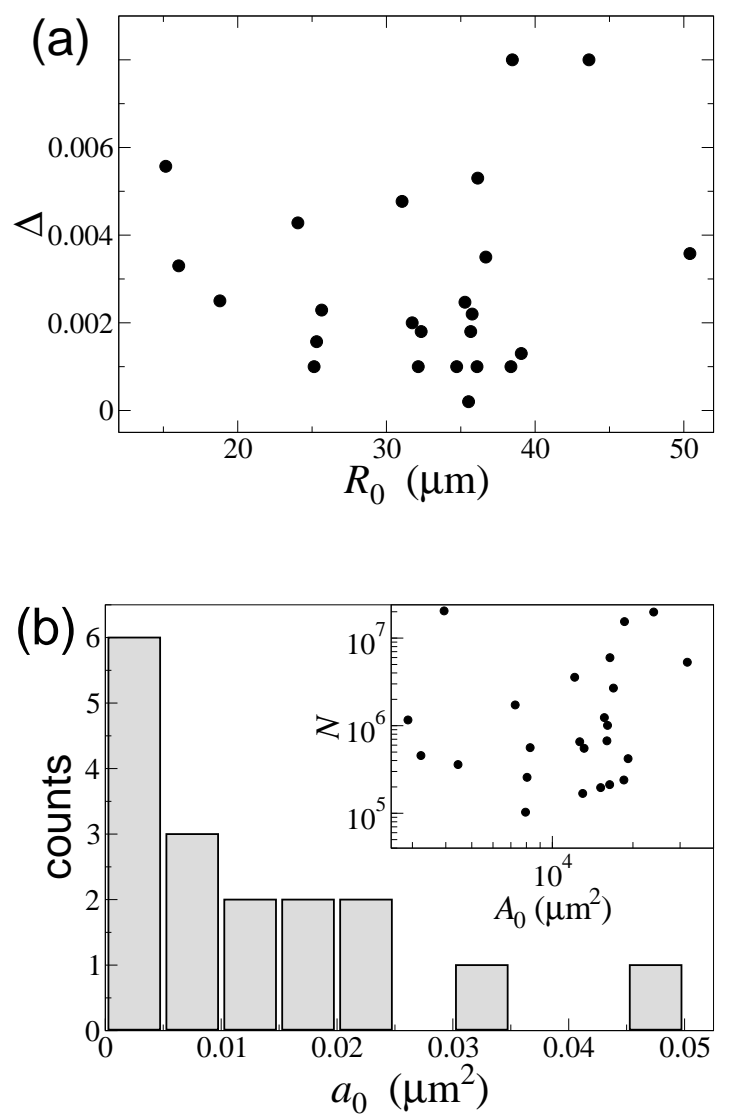

FIG. 7. (a) Transition width vs. vesicle radius, exhibiting no correlation between the two variables. (b) Histogram of effective patch size values, obtained from the measured $\Delta$ of panel (a) using Eq. (18) and $K=240 \mathrm{mN} / \mathrm{m}$. Inset shows the resulting number of effective bending modes vs. vesicle area.

\section{DISCUSSION}

The experimental results presented above confirm the critical nature of the osmotic swelling of vesicles, as was first suggested in Ref. 7. It is important to note that a similar critical scaling is absent in the case of hydrostatic swelling, as in vesicle aspiration experiments [8]. To adapt our formulation to such a case we should merely replace the solute contribution to the Gibbs free energy (the first term in Eq. (44)), with $\left(-p_{\text {in }} V\right)$, where $p_{\text {in }}$ is the inner hydrostatic pressure. Repeating the minimization procedure presented in Sec. IV] we find that Eq. (12) remains unchanged, while the swelling order parameter becomes

$$
M=1-v=\frac{\delta_{N}}{2\left(p_{\text {in }} / p_{\mathrm{o}}-1\right)} .
$$

Thus, our formalism applies in this case only for sufficiently large inner pressures, $p_{\text {in }} / p_{\mathrm{o}}>1+\delta_{N} / 2$ (to ensure a positive $v$ ). Similar to $q=Q / Q_{\mathrm{c}}-1$, we may define a swelling control parameter, $q^{\prime}=p_{\text {in }} / p_{\mathrm{o}}-1$, yet its value is restricted to $q^{\prime}>\delta_{N} / 2$. Consequently, Eq. (22) yields a moderate decrease of $M$ from appreciable to small values, $M \sim 1 / q^{\prime}-i . e .$, a gradual approach to a spherical shape. Another way to demonstrate the non-criticality of the hydrostatic-pressure case is to recognize that, once both $p_{\mathrm{o}}$ and $p_{\text {in }}$ are specified, the surface tension of a nearly spherical vesicle is simply given by Laplace's law rather than the more elaborate critical expressions of Eqs. (14)(16). This underlines the special thermodynamic behavior of osmotically stressed vesicles, as was indicated before in a more general statistical-mechanical context [28]. The key difference between osmotically swollen and directly pressurized vesicles lies in the fact that the pressure difference of the former depends on the volume for a given number of encapsulated solute molecules [cf. Eq. (4)], whereas the pressure difference of the latter is a given constraint. This makes the statistical ensembles of $\left(T, p_{\mathrm{o}}, Q, N\right)$ and $\left(T, p_{\mathrm{o}}, p_{\text {in }}, N\right)$ not equivalent [28].

We have shown how the data collapse for different vesicle sizes can be utilized to obtain a sensitive measurement of the membrane permeability coefficients of various solutes. This ability is particularly well demonstrated in the case of urea (Fig. 6(a)). Overall, the permeability values that we have obtained are in accord with previously published ones. (See the detailed account in the preceding section and Fig. 6(b).) In addition, we have found an increasing concentration dependence of the permeability for glycerol and ethylene glycol. While we are not aware of an earlier report of such an effect, it is in line with strong evidence for the affinity of polyols to lipid headgroups [29, 30] and may account for the large range of values reported in the literature for the permeability coefficients of these solutes. The affinity should make the solute concentration adjacent to the membrane larger than in the bulk solution, leading to an apparent permeability coefficient which is larger than the actual one. More extensive measurements of the apparent per- 
meability as a function of concentration might allow an extraction of the solute-membrane affinity parameters. At higher concentrations the solute may also disrupt the membrane structure, thus affecting the actual permeability 31] and possibly other membrane properties [32].

The transition width, and the corresponding effective membrane patch $a_{0}$, have been found to be broadly distributed (Fig. 7). The origin of this stochasticity is not yet clear. It may be that $a_{0}$ is sensitive to the presence of membrane impurities. Nevertheless, the analysis of transition widths has enabled us to obtain information regarding the number of independent bending modes, $N$, which contribute to the thermodynamics of the vesicle in the transition region. To our knowledge this variable has not been experimentally accessed before. For the vesicles used here, having $R_{0} \simeq 20-50 \mu \mathrm{m}$, we have found numbers $N \sim 10^{5}-10^{7}$, which are large but much smaller than the number of lipid molecules in the vesicle (about $\left.10^{10}\right)$.

To account for these intermediate values of $N$ we should link our thermodynamic formulation to the more detailed statistical mechanics of fluctuating vesicles. The definition of $N$ in Sec. [V] has been through the surface entropy of a nearly spherical vesicle, Eq. (7), where each of the $N$ modes contributes an equal amount of $\left(k_{\mathrm{B}} T / 2\right)|\ln (1-v)|$ to the free energy [7]. Examining more closely the partition function of a fluctuating vesicle within Helfrich's model [10], we find that these modes, whose contribution is singular in $(1-v)$, belong to the small-wavenumber (tension-dominated) portion of the fluctuation spectrum. Their number is

$$
N \simeq \gamma R_{0}^{2} / \kappa
$$

where $\kappa$ is the membrane's bending rigidity. Equations (15), (16), and (23) then allow a theoretical estimate of $N$. At the transition we have $\gamma \simeq(2 / 3) K \Delta \tilde{M}(-1)$ (cf. Fig. 5), which for $K \simeq 240 \mathrm{mN} / \mathrm{m}$ [24] and $\Delta \simeq 0.004$ (Fig. 7(a)) yields $\gamma \simeq 0.8 \mathrm{mN} / \mathrm{m}$. This value of about 1 $\mathrm{mN} / \mathrm{m}$ is in line with that obtained for nearly spherical vesicles in aspiration experiments [8]. Substituting it, together with $\kappa \simeq 10 k_{\mathrm{B}} T 24$ and $R_{0} \simeq 20-50 \mu \mathrm{m}$, in Eq. (23), we get $N \sim 10^{7}$, which is in the range of values that we have found experimentally.

Equation (23) implies, in fact, that $N$ is not constant but increases with the degree of swelling (i.e., with $\gamma$ ). Hence, sufficiently far below the transition $\gamma$ will be too small, and the theory should fail. (Far beyond the transition, on the other hand, the behavior is dominated by surface strain and unaffected by these entropy considerations.) Combining Eqs. (16) and (23), we find that the theory should break down for $M \gtrsim 3 k_{\mathrm{B}} T /(16 \pi \kappa) \simeq$ 0.006 . With $\Delta \simeq 0.004$ this occurs for $x \lesssim-1.3$, which is consistent with the region where the data collapse begins to fail (see Fig. [5).

Thus, the assumption of a swelling-independent $N$ is the main limitation of the model and should be improved in future studies. Other assumptions are less significant. For example, the relaxed area of the vesicle, $A_{0}$, is actu- ally slightly smaller than the assumed value of $4 \pi R_{\min }^{2}$, where $R_{\text {min }}$ is the minimum of the experimental $R_{1}(t)$ curve. The calculation given in the Appendix implies that $R_{0} / R_{\min } \simeq 1-\left(15^{1 / 3} / 4\right) \Delta^{2 / 3}$. For our broadest transitions this deviation amounts to less than $3 \%$. As shown in Ref. 7, accounting for additional factors such as a non-ideal solute does not affect the universal behavior in the vicinity of the transition.

\section{Acknowledgments}

We thank Oded Farago, Rony Granek, and Luka Pocivavsek for helpful comments. EH and HD acknowledge the Donors of the American Chemical Society Petroleum Research Fund for support of this research (Grant No. 46748-AC6).

\section{APPENDIX}

In this appendix we adapt the theoretical scaling relation obtained in Sec. IV to the observables in the experimental swelling curves. The result is the scaling transformation that should be applied to the experimental data to achieve data collapse.

Consider an oblate spheroid of principal radii $R_{1}$ and $R_{2}<R_{1}$, and small eccentricity $e=\left[1-\left(R_{2} / R_{1}\right)^{2}\right]^{1 / 2} \ll$ 1. The volume of the spheroid is $V=(4 \pi / 3) R_{1}^{2} R_{2}$, and its area is $A=\pi\left\{2 R_{1}^{2}+\left(R_{2}^{2} / e\right) \ln [(1+e) /(1-e)]\right\}$. From this we get $v \simeq 1-e^{4} / 15+O\left(e^{6}\right)$, leading to $e^{2} \simeq(15 M)^{1 / 2}+O(M)$, and $R_{2} / R_{1}=\left(1-e^{2}\right)^{1 / 2} \simeq$ $1-(15 M)^{1 / 2} / 2+O(M)$. Now we have, on the one hand, $V / V_{0}=\left(A / A_{0}\right)^{3 / 2} v=(1+\epsilon)^{3 / 2} v$, and, on the other hand, $V / V_{0}=\left(R_{1} / R_{0}\right)^{3}\left(R_{2} / R_{1}\right)$. Equating these two expressions while using the result above for $R_{2} / R_{1}$, we obtain

$$
\left(R_{1} / R_{0}\right)^{3}-1 \simeq(15 M)^{1 / 2} / 2+3 \epsilon / 2 .
$$

The correction to this expression is $O(M)$. In the transition region this correction, as well as $\epsilon$, are negligible $(\sim \Delta)$ compared to the $M^{1 / 2}$ term $\left(\sim \Delta^{1 / 2}\right)$. Above the transition, at the point where $\epsilon$ becomes comparable to $M^{1 / 2}$, we find $\epsilon \sim M^{1 / 2} \sim \Delta^{2 / 3}$, whereas $M \sim \Delta^{4 / 3}$. Thus, Eq. (A1) contains the leading terms within the transition region as well as above it. Using Eqs. (14), (15), and the property $\tilde{M}(-x)=\tilde{M}(x)+x$, we rewrite Eq. (A1) as

$$
\begin{aligned}
& \left(R_{1} / R_{0}\right)^{3}-1 \simeq(15 \Delta / 8)^{1 / 2} f(q / \Delta)+q \\
& f(x)=\left(\sqrt{1+x^{2}}-x\right)^{1 / 2},
\end{aligned}
$$

where we have omitted another term of order $M$ and also the ratio $\delta_{N} / \delta_{K}$.

Equation (A2), as required, contains the experimental observable $R_{1} / R_{0}$ rather than our order parameter $M$. Yet, we need to take one last step before we can apply it to the experimental data. The expression in Eq. 
(A2) has a minimum at $\left[q \simeq\left(15^{1 / 3} / 4\right) \Delta^{2 / 3},\left(R_{1} / R_{0}\right)^{3}-\right.$ $\left.1 \simeq 3\left(15^{1 / 3} / 4\right) \Delta^{2 / 3}\right]$, which is shifted from the one that we have defined for the experimental curve, $[q=$ $0,\left(R_{1} / R_{0}\right)^{3}-1=0$ ] (cf. Fig. 1). In other words, the actual relaxed radius is slightly shifted from the minimum of the measured swelling curve. To correct for this $\sim \Delta^{2 / 3}$ error, we ought to shift the experimental curves accordingly. If $\left[R_{1}(t) / R_{0}\right]^{3}-1=g_{\exp }(t)$ is the measured dependence, as a function of $q$, then the following scaling and shifting transformation,

$$
f(x)=(15 \Delta / 8)^{-1 / 2}\left\{g_{\exp }\left[x-\left(15^{1 / 3} / 4\right) \Delta^{-1 / 3}\right]-x \Delta\right.
$$

$$
\begin{aligned}
& \begin{array}{l}
+3\left(15^{1 / 3} / 4\right) \Delta^{2 / 3} \\
x=q / \Delta, \quad q=\left(3 P / R_{0}\right) t
\end{array}
\end{aligned}
$$

should collapse the data onto the universal function $f(x)$ defined in Eq. (A2). This is the transformation used in Sec. V.
[1] S. A. Safran, Statistical Thermodynamics of Surfaces, Interfaces, and Membranes, Addison Wesley, 1994.

[2] U. Seifert, Adv. Phys., 1997, 46, 13-137.

[3] H. Lodish et al., Molecular Cell Biology, 6th edition, Freeman, 2008.

[4] D. D. Lasic, Liposomes: From Physics to Applications, Elsevier, 1993.

[5] S. Paula, A. G. Volkov, A. N. Van Hoek, T. H. Haines and D. W. Deamer, Biophys. J., 1996, 70, 339-348.

[6] B. L.-S. Mui, P. R. Cullis, E. A. Evans and T. D. Madden, Biophys. J., 1993, 64, 443-453.

[7] E. Haleva and H. Diamant, Phys. Rev. Lett., 2008, 101, 078104.

[8] E. Evans and W. Rawicz, Phys. Rev. Lett., 1990, 64, 2094-2097.

[9] W. Helfrich and R.-M. Servuss, Nuovo Cimento, 1984, 3, 137-151.

[10] S. T. Milner and S. A. Safran, Phys. Rev. A, 1987, 36, 4371-4379.

[11] M. I. Angelova and D. S. Dimitrov, Faraday Discuss. Chem. Soc., 1986, 81, 303-311.

[12] V. Heinrich and R. E. Waugh, Ann. Biomed. Eng., 1996, 24, 595-605.

[13] P. Peterlin and V. Arrigler, Colloid Surf. B, 2008, 64, 77-87.

[14] R. Dimova, S. Aranda, N. Bezlyepkina, V. Nikolov, K. A. Riske and R. Lipowksy, J. Phys. Condens. Matter, 2006, 18, S1151-S1176.

[15] P. Peterlin, G. Jaklič and T. Pisanski, Meas. Sci. Technol., 2009, 20, 055801.

[16] H.-G. Döbereiner, E. Evans, M. Kraus, U. Seifert and M.
Wortis, Phys. Rev. E, 1997, 55, 4458-4474.

[17] G. T. Linke, R. Lipowsky and T. Gruhn, Phys. Rev. E, 2005, 051602.

[18] J. D. Litster, Phys. Lett., 1975, 53A, 193-194.

[19] O. Farago and C. D. Santangelo, J. Chem. Phys., 2005, 122, 044901.

[20] H. Diamant, Phys. Rev. E, 2011, 84, 061123.

[21] O. Farago and P. Pincus, J. Chem. Phys., 2004, 120, 2934-2950.

[22] J.-B. Fournier and C. Barbetta, Phys. Rev. Lett., 2008, 100, 078103.

[23] F. Schmid, EPL, 2011, 95, 28008.

[24] D. Marsh, Chem. Phys. Lipids, 2006, 144, 146-159.

[25] M. Mally, J. Majhenc, S. Svetina and B. Žekš, Biophys. J., 2002, 83, 944-953.

[26] C. Dordas and P. H. Brown, J. Membr. Biol., 2000, 175, 95-105.

[27] E. Orbach and A. Finkelstein, J. Gen. Physiol. 1980, 75, 427-436.

[28] E. Haleva and H. Diamant, Phys. Rev. E, 2008, 75, 021132 .

[29] N. Krasteva, D. Vollhardt, G. Brezesinski and H. Mohwald, Langmuir, 2001, 17, 1209-1214.

[30] L. Pocivavsek, K. Gavrilov, K. D. Cao, E. Y. Chi, D. Li, B. Lin, M. Meron, J. Majewski and K. Y. C. Lee, Biophys. J., 2011, 101, 118-127.

[31] H. Patel, G. Raval, M. Nazari and H. Heerklotz, Biophys. Chem., 2010, 150, 119-128.

[32] V. Vitkova, J. Genova, M. D. Mitov and I. Bivas, Mol. Cryst. Liq. Cryst., 2006, 449, 95-106. 